\title{
Etude Phytochimique de Senna occidentalis (L.) Link et Cissus quadrangularis (Linn) deux Plantes Médicinales Gabonaises Utilisées Contre la Filaire Loa Loa
}

\section{Leaticia Nzé Kamsi (Master Professionnel des Sciences de la Vie et de la Terre)}

Laboratoire d'Immunologie et de Microbiologie Appliquée Ecole Normale Supérieure (ENS), Libreville, Gabon

\section{Line Edwige Mengome (Docteur en Biologie des Substances Naturelles)}

Institut de Pharmacopée et Médecine Traditionnelle (IPHAMETRA), Centre

National de la Recherche Scientifique et Technique (CENAREST),

Département de Phytochimie, Libreville, Gabon

Sophie Aboughe-Angone (Docteur en Biologie des substances naturelles)

Institut de Pharmacopée et Médecine Traditionnelle (IPHAMETRA), Centre

National de la Recherche Scientifique et Technique (CENAREST),

Département de Phytochimie, Libreville, Gabon

Prospert Edou Engonga (Docteur en Chimie Analytique)

Laboratoire de Chimie Organique et Analytique, Ecole Normale Supérieure (ENS), Libreville, Gabon

Doi:10.19044/esj.2020.v16n21p101 URL:http://dx.doi.org/10.19044/esj.2020.v16n21p101

Résumé

Objectif : Cette étude avait pour objectif de valoriser deux plantes médicinales utilisées au Gabon pour traiter la filaire Loa loa. Matériels et Méthodes : Les poudres des feuilles de Senna occidentalis et des tiges et feuilles de Cissus quadrangularis ont été soumis à une succession d'extractions par des solvants de polarités croissantes (cyclohexane, dichlorométhane, méthanol et l'eau déminéralisée). Un criblage phytochimique qualitatif a été effectué en utilisant la méthode standard basée sur des réactions de colorations et/ou de précipitations. Résultats : les résultats révèlent la prédominance des sucres totaux, des alcaloïdes, et des phénols dans tous les extraits. Les des tanins cathéchiques, les sucres réducteurs, et les flavonoïdes sur de nombreux extraits testés. Les tanins galliques et les 
coumarines sont retrouvés dans quelques extraits. Très peu de quinones, de triterpènes ou stérols dans les deux plantes. Conclusions : Certains métabolites secondaires ont déjà prouvé leurs activités filaricides. La richesse en composés chimiques présents dans ces plantes médicinales étudiées, justifierait leur utilisation. Cependant, l'évaluation des activités biologiques sur la filariose à Loa loa ainsi que leur toxicité reste à explorer.

Mots-clés : Criblage Phytochimique, Plantes Médicinales, Filaire Loa Loa, Gabon

\section{Phytochemical Study of Senna occidentalis (L.) Link and Cissus quadrangularis (Linn) of Two Gabonese Medicinal Plants used Against Loa Loa Filarial}

\section{Leaticia Nzé Kamsi (Master Professionnel des Sciences de la Vie et de la Terre)}

Laboratoire d'Immunologie et de Microbiologie Appliquée Ecole Normale Supérieure (ENS), Libreville, Gabon

Line Edwige Mengome (Docteur en Biologie des Substances Naturelles)

Institut de Pharmacopée et Médecine Traditionnelle (IPHAMETRA), Centre

National de la Recherche Scientifique et Technique (CENAREST),

Département de Phytochimie, Libreville, Gabon

Sophie Aboughe-Angone (Docteur en Biologie des substances naturelles)

Institut de Pharmacopée et Médecine Traditionnelle (IPHAMETRA), Centre

National de la Recherche Scientifique et Technique (CENAREST),

Département de Phytochimie, Libreville, Gabon

Prospert Edou Engonga (Docteur en Chimie Analytique)

Laboratoire de Chimie Organique et Analytique, Ecole Normale Supérieure (ENS), Libreville, Gabon

\footnotetext{
Abstract

Objective: This study aimed to valorize two medicinal plants used against Loa loa filarial in Gabon. Material and Methods: Powders of leaves of Senna occidentalis and stems and leaves of Cissus quadrangularis were proposal to successive extractions by solvents with increasing polarities
} 
(cyclohexan, dichloromethan, methanol and demineralized water). Qualitative Phytochemistry screening was established using the standard color and/or precipitation reactions. Results: The results reveal the predominance of total sugar, alkaloids, and phenols in all extracts. Cathechic tannins, reducing sugar, and flavonoids on many extracts were tested. Gallic tannins and coumarins are found in some extracts. They are very few quinones, triterpenes or sterols in the two species. Conclusion: Some secondary metabolites have proved filaricide activities already. The abundance of chemical compounds present in these medicinal plants would justify their usage. However, the evaluation of biological activities on the Loa loa filarial along with toxicity remains unexplored.

Keywords: Phytochemistry Screening, Medicinal Plants, Loa Loa Filarial, Gabon

\section{Introduction}

Les filaires Loa loa sont des nématodes du groupe des vers ronds endémiques des forêts pluviales et tropicales de l'Afrique Centrale, de l'Ouest et Occidentale (Zouré et al., 2011). La filaire Loa loa est transmise par une mouche de la famille des tabanidées du genre Chrysops (Chrysops silacea, Chrysops dimidiata ou Chrysops distinctipennis) (Boussinesq, 2006 ; Antinori et al., 2012). Au Gabon, la prévalence de la filaire Loa loa est de 22,4\% et se répartie dans différents écosystèmes comme suit: 14,8\% dans les savanes, 17 $\%$ dans les lacs, 20,5\% dans les montagnes, 20,6\% dans les forêts du nordest, 24,3\% dans les forêts et de 28,9\% dans les prairies (Akué et al., 2011). L'Organisation Mondiale de la Santé (OMS) estime qu'environ 300 millions de personnes sont à risque et 13 millions sont infectées (OMS, 2018). Dans les zones d'endémicité de la filariose à Loa loa, c'est la troisième raison de consultation après le paludisme et les maladies pulmonaires (Mengome et al., 2010 ; Akué et al., 2011). Cette infection touche aussi bien les populations autochtones que les expatriés qui y vivent (Fain, 1981). Bien que non mortelle à court terme, la filariose à Loa loa peut devenir une maladie systémique à cause de sa chronicité et atteindre des organes tels que le cour, les reins, le foie, les poumons, elle peut aussi entraîner un dérèglement de la réponse immunitaire de l'hôte (Fain, 1981). Le traitement des filaires présente une bonne sensibilité à la Diéthylcarbamazine (DEC) et à l'ivermectine (IVM). Cependant, ces deux médicaments sont surtout actifs sur les microfilaires et très peu sur les vers adultes (Newman \& Cragg, 2007). Ces médicaments induisent également des effets secondaires tels que les troubles gastrointestinaux, les maux de têtes, les éruptions cutanées, des hémorragies rétiniennes (Moussala et al., 2004; Behera \& Bhatnagar, 2018). Le programme de masse de lutte contre l'onchocercose et/ou la filariose 
lymphatique sur des patients contenant un fort taux de Loa loa environ 8000 microfilaires par millilitre de sang peut provoquer un choc anaphylactique (Chippaux et al., 1996 ; Ndjonka et al., 2013). Aussi, les maladies débilitantes (œdèmes de Calmar, prurits, migrations aliphatiques du ver adulte) qu'induisent Loa loa entrainent un frein dans la production agricole en zone rurale (Agbolade et al., 2005). Le vecteur n'étant pas éradiqué lors de la lutte contre cette filariose, on peut observer une réinfection dans les zones d'endémicités. Dans les pays en voie de développement, les problèmes de médicaments se posent en terme d'insuffisance quantitative, qualitative et d'inaccessibilité économique (Pawlaczyk et al., 2011). Ces pertinences font de la filaire Loa loa un problème de santé publique. Des traitements alternatifs viendront en à point pour la recherche de nouveaux médicaments efficaces, tolérés, accessibles et ayant peu d'effets indésirables.

Selon Lengani et al. (2010), l'OMS estime que $40 \%$ des médicaments ont comme matière active une substance naturelle et qu'environ $80 \%$ de la population mondiale à recourt aux plantes pour se soigner. L'étude phytochimique des plantes médicinales peut donc contribuer à la réglementation de l'usage empirique, des modes de consommation avec une efficacité scientifiquement prouvée et une acceptabilité culturelle optimale. Dans le monde, des études pharmacologiques sur des extraits de plantes, des métabolismes secondaires, des principes actifs ou des biomolécules ont prouvés leur efficacités sur des parasites, des larves, etc. Plusieurs travaux ont été faits sur les filaires lymphatiques ainsi que sur l'onchocercose, mais très peu l'ont été sur la filaire Loa loa. La présente étude porte sur le criblage phytochimique des extraits aqueux, méthanoliques, cyclohexaniques et au dichlorométhane des feuilles de Senna occidentalis puis des tiges et des feuilles de Cissus quadrangularis deux plantes médicinales utilisées au Gabon contre la filaire Loa loa.

\section{Matériels et Méthodes}

\section{Matériels}

\subsection{Présentation des végétaux}

Senna occidentalis (a) ou faux kinkéliba est un sous arbuste de la famille des Fabaceae, sous-famille des Caesalpinioides selon la classification phylogénique des végétaux. Il a pour synonyme Cassia occidentalis L., c'est une plante originaire de l'Amérique du Sud qui est devenue une espèce pantropicale. Ces feuilles sont persistantes, lancéolées, composées et brillantes à la surface des feuilles. Ses racinaires sont profondes, sa tige est dure et ligneuse, ces graines sont dicotilédonnes avec une odeur caractéristique et un goût amer. Ces fleurs sont de couleur jaune avec un diamètre de 1 à $2 \mathrm{~cm}$ disposées en inflorescence de type racème, axillaires et également terminales, 
les bractées sont caducées. Les fruits sont des gousses plates de 10 à $12 \mathrm{~cm}$ de long avec 10 à 30 graines pointues à l'extrémité.

Cissus quadrangularis (b) est une espèce végétale appartenant à la famille des Vitaceae, sous-famille des Vitoïdeae probablement originaire de l'Inde et du Sri Lanka, il est étendu en Afrique, en Arabie, dans le sud-est de l'Asie, ainsi que dans le sud des Etats-Unis et au Brésil où il a été importé. Il a pour synonymes Cissus quadrangula Salisb., et Vitis quadrangularis (L.) Wall. ex Wight. C'est une plante grimpante vivace pouvant atteindre plusieurs mètres de longueurs. Ces feuilles caduc sont alternes, lobées, dentelées et palmées. Sa tige carrée, presque ailées, trapues et glabes, stipules ovales, atteignant 5 $\mathrm{mm}$ de long; pétiole de 5-11 mm de long ; limbe un peu charnu, ovale ou largement ovale. Ces fleurs rosées à l'intérieur, verts et rouges à l'extérieur, parfumées peuvent atteindre 2,5 mm de long. Ces Fruits à baie arrondie, charnues, atteignent $12 \mathrm{~mm}$ de diamètre, jaunes à rouges ou violettes, calice et style persistants, à 1 graine.
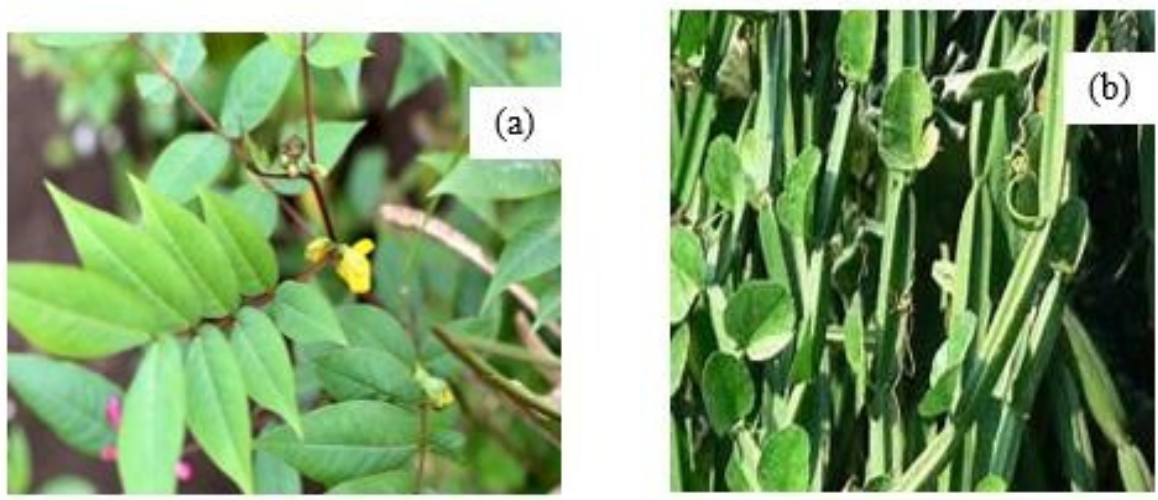

Figure 1. Images illustrant des tiges feuillées de Senna occidentalis (L.) Link. (Caesalpiniaceae) (a) et de Cissus quadrangularis (Linn) (Vitaceae) en (b).

\subsection{Appareils et outils}

Déshumidificateur (Bioblock Scientific LMS Cooled Incubator), broyeur (Broyeurs à percussion RETSCH ${ }^{\circledR}$ ), balance, rotavapore (BUCHI Rotavapor $\left.{ }^{\mathrm{TM}} \mathrm{R}-100\right)$, lampe UV, étuve, bain-marie, barreaux aimantés, béchers de $500 \mathrm{ml}$, Erlen mayer de $200 \mathrm{ml}$, pro-pipette, pipettes graduées.

\subsection{Solvants et réactifs}

Les solvants utilisés sont: le cyclohexane, le dichlorométhane, le méthanol et de l'eau déminéralisée. Le réactif de Dragendorff, de Mayer, de Fehling (Carlo Erba, Prolabo), de Molish, l'acide chlorhydrique, l'acide sulfurique, l'anhydride acétique, l'ammoniaque $30 \%$, l'alcool isoamylique, l'hydroxyde de sodium, le chlorure ferrique, et les fragments de magnésium (Sigma) ont été utilisé comme réactifs. 


\section{Méthodes d'études phytochimiques}

\subsection{Préparation des échantillons}

Les plantes étudiées sont obtenues au cours des enquêtes ethnobotaniques faites par le département de médecine traditionnelle de l'IPHAMETRA. Ces plantes ont été récoltées au Cap Estériase par l'équipe de botanistes du département de l'Herbier National du Gabon (HNG), sous les références (3237B) pour $S$. occidentalis et (125) pour C. quadrangularis. Caractérisées puis mises à sécher à l'aide d'un déshumidificateur à $35^{\circ} \mathrm{C}$ pendant une semaine. Les échantillons sont découpées en fines particules à l'aide d'un sécateur puis réduites en poudre à l'aide d'un broyeur électrique. Ces échantillons sont codifiés comme suit: les feuilles de Cissus quadrangularis $(\mathbf{C q} \mathbf{f})$; les feuilles de Senna occidentalis (Sof) enfin, les tiges de Cissus quadrangularis $\left(\mathbf{C q}_{\mathbf{t}}\right)$.

\subsection{Préparation des extraits}

Quatre types d'extraits ont été préparés pour chaque partie de plantes étudiées : trois extraits organiques et un extrait aqueux. A cet effet, $10 \mathrm{~g}$ de poudre de chaque échantillon ont été pesés puis soumis à une succession d'extractions par des solvants de polarité croissante. Chaque poudre est mélangée à $100 \mathrm{ml}$ de cyclohexane puis laissée macérer pendant 1 heure.

Après filtration, l'extrait cyclohexanique (EC) obtenu est conservé. Le résidu séché à température ambiante est repris dans $100 \mathrm{ml}$ de dichlorométhane pendant 1 heure, après filtration, l'extrait au dichlorométhanique (ED) obtenu est conservé. Le résidu séché est mélangé dans $100 \mathrm{ml}$ de méthanol pendant 24 heures. Le filtrat nous permet d'obtenir l'extrait méthanolique (EM). Quant à l'extrait aqueux (EA), $10 \mathrm{~g}$ de poudre de chaque drogue sont mise à ébullition avec $100 \mathrm{ml}$ d'eau déminéralisée dans un bain-marie à $100^{\circ} \mathrm{C}$ pendant 1 heure, puis filtré après refroidissement. L'ensemble des extraits obtenus ont été soumis à un criblage phytochimique selon le protocole de notre laboratoire. La liste des extraits obtenus est représentée dans le Tableau 1. 


\begin{tabular}{|c|c|c|c|}
\hline $\begin{array}{l}\text { Noms scientifiques / } \\
\text { Familles / } \mathbf{N}^{\circ} \text { herbier }\end{array}$ & $\begin{array}{l}\text { Parties } \\
\text { utilisées }\end{array}$ & Solvants & Extraits \\
\hline \multirow{8}{*}{$\begin{array}{l}\text { Cissus Quadrangularis } \\
\text { (Linn) / Vitaceae / } 125\end{array}$} & \multirow{4}{*}{ Feuilles $\left(\mathrm{Cq}_{\mathrm{f}}\right)$} & Cyclohexane & $\mathrm{Cq}_{\mathrm{f}}-\mathrm{EC}$ \\
\hline & & Dichlorométhane & $\mathrm{Cq}_{\mathrm{f}}-\mathrm{ED}$ \\
\hline & & Méthanol & $\mathrm{Cq}_{\mathrm{f}}-\mathrm{EM}$ \\
\hline & & Eau déminéralisée & $\mathrm{Cq}_{\mathrm{f}}-\mathrm{EA}$ \\
\hline & \multirow{4}{*}{ Tige $\left(\mathrm{Cq}_{\mathrm{t}}\right)$} & Cyclohexane & $\mathrm{Cq}_{\mathrm{t}}-\mathrm{EC}$ \\
\hline & & Dichlorométhane & $\mathrm{Cq}_{\mathrm{t}}-\mathrm{ED}$ \\
\hline & & Méthanol & $\mathrm{Cq}_{\mathrm{t}}-\mathrm{EM}$ \\
\hline & & Eau déminéralisée & $\mathrm{Cq}_{\mathrm{t}}-\mathrm{EA}$ \\
\hline \multirow{4}{*}{$\begin{array}{l}\text { Senna Occidentalis } \\
\text { (Link) / } \\
\text { Caesalpinioceae / } \\
\text { 3237B }\end{array}$} & \multirow{4}{*}{ Feuilles $\left(\mathrm{So}_{\mathrm{f}}\right)$} & Cyclohexane & $\mathrm{So}_{\mathrm{f}}-\mathrm{EC}$ \\
\hline & & Dichlorométhane & $\mathrm{So}_{\mathrm{f}}-\mathrm{ED}$ \\
\hline & & Méthanol & So $_{f}-E M$ \\
\hline & & Eau déminéralisée & $\mathrm{So}_{\mathrm{f}}-\mathrm{EA}$ \\
\hline
\end{tabular}

Tableau 1. Identification des extraits

\subsection{Criblage phytochimique:}

Les méthodes standards précédemment décrites par Mengome et al., (2009) et Feuya et al., (2015) ont été utilisées avec quelques modifications. Les groupes chimiques que nous avons identifiés sont les alcaloïdes, les tanins, les flavonoïdes, les quinones, les stérols et triterpènes, les caroténoïdes, les sucres totaux, les sucres réducteurs, les coumarines et les saponosides.

Test des sucres totaux : A $2 \mathrm{ml}$ de chaque extrait sont ajouté 3 gouttes de réactif de Molish puis $1 \mathrm{ml} \mathrm{d}$ 'acide sulfurique concentré. L'apparition d'une interphase de coloration violette indique la présence des sucres totaux.

Test des sucres réducteurs : La méthode réductrimétrique de Fehling a été utilisée. $2 \mathrm{ml}$ d'eau déminéralisée sont ajutés aux $2 \mathrm{ml}$ de chaque extrait organique contenu dans chaque tube puis, $1 \mathrm{ml}$ de réactif de liqueur de Fehling y est ajouté. Ces tubes ont été portés à ébullition pendant 30 minutes. La formation d'un précipité rouge-brique indique la présence des composés réducteurs.

Test des alcaloïdes : Deux types de tests ont été effectués. Pour le test de Mayer. 1,5 $\mathrm{ml}$ d'acide chlorhydrique $2 \%$ sont ajoutés dans chaque tube, puis 2 à 3 gouttes du réactif de Mayer sont ajoutés. La formation d'un précipité blanc témoigne de la présence d'alcaloïdes. Pour le test de Dragendorff $2 \mathrm{ml}$ d'éthanol $60^{\circ} \mathrm{C}$ sont ajoutés dans chaque tube en agitant avec une petite baguette de verre au bain-marie, puis 2 à 3 gouttes du réactif de Dragendorff sont ajoutés. La présence d'un précipité rouge ou d'une coloration orangée indique la présence d'alcaloïdes.

Test des tanins : $2 \mathrm{ml}$ d'eau déminéralisée sont ajoutés à $2 \mathrm{ml}$ de chaque extrait. Ensuite 5 gouttes de solution de chlorure ferrique à $1 \%$ y sont ajoutées. La présence de tanins galliques est caractérisée par le développement d'une teinte bleu-noire et celle des tanins cathéchiques par une teinte brunverdâtre. 
Test des stérols ou triterpènes : $1 \mathrm{ml}$ d'anhydride acétique est ajouté à $2 \mathrm{ml}$ de chaque extrait. Par la suite, $1 \mathrm{ml}$ d'acide sulfurique concentré est coulé dans les tubes, c'est la réaction de Liebermann-Buchard. L'apparition d'une interphase pourpre ou violet et la coloration du surnageant vert bleu ou violet indique respectivement la présence de stérols ou de triterpènes.

Test des flavonoïdes (réaction à la cyanidine) : $2 \mathrm{ml}$ de chaque extrait a été repris dans $1 \mathrm{ml}$ de méthanol à $50 \%$ au bain-marie. 2 à 3 fragments de copeau de magnésium et 4 à 5 gouttes d'acide chlorhydrique concentré 99,9 $\%$ y sont ajoutés. Le dégagement de chaleur puis l'apparition d'une coloration rose-orangée indique la présence des flavones, rose-violacée celle des flavonones et rouge cerise pour les flavanols.

Test des caroténoïdes : $0,5 \mathrm{ml}$ d'acide sulfurique concentré est ajouté à $2 \mathrm{ml}$ de chaque échantillons. L'apparition d'une coloration bleue qui vire au rouge indique la présence des caroténoïdes.

Test des coumarines : $1 \mathrm{ml}$ d'ammoniaque $20 \%$ est ajouté dans chaque extrait. L'ensemble est chauffé au bain-marie pendant 5 minutes puis une lecture en UV à $365 \mathrm{~nm}$ est effectuée. L'apparition d'une fluorescence (jaune, bleue, bleue-vert, orange, rose violace) indique la présence de coumarines.

Test des quinones : Avec la réaction de Bornstraegen, $2 \mathrm{ml}$ de chaque extraits sont repris dans $1 \mathrm{ml}$ d'acide chlorhydrique concentré $99,9 \%$ puis chauffés au bain-marie pendant 15 minutes, après refroidissement, $2 \mathrm{ml}$ de chloroforme sont ajoutés puis $2 \mathrm{ml} \mathrm{d}$ 'ammoniaque $20 \%$ et 5 gouttes de soude $10 \%$. L'apparition d'une coloration rouge ou violette indique la présence des quinones.

Test des saponines : Dans une série de 10 tubes à essai sont repartis successivement 1 à $10 \mathrm{ml}$ de l'extrait aqueux. Puis chaque tube est complété pour un volume total de $10 \mathrm{ml}$ avec de l'eau déminéralisée. Les tubes sont agités vigoureusement dans le sens de la longueur pendant 15 secondes puis laissés au repos. Après $15 \mathrm{~min}$ la hauteur de la mousse $(\mathrm{Hm})$ est mesurée afin de calculer l'indice de mousse (IM) dont la formule est la suivante $\mathrm{IM}=1000$ / Hm. Le tube dans lequel la hauteur de mousse est de $1 \mathrm{~cm}$ indique la valeur de l'index de mousse.

\section{Résultats}

\section{Contenu qualitatif des plantes étudiées}

Les résultats du criblage phytochimique sont présentés dans le Tableau 2. Le signe (+) indique une réaction positive et traduit la présence du groupe de composés recherchés tandis que le signe (-) indique une réaction négative. L'intensité de la coloration observée a été assimilée à la proportion de chaque composé et traduite sur une échelle allant de un à trois par des signes $(+),(++)$ et $(+++)$. 
L'incidence des résultats positifs présentée dans la dernière colonne du Tableau 2 est effectuée selon la formule suivante: pourcentage de résultats positifs $=$ (résultats positifs de chaque extrait / nombre total de tests par extrait) x 100 . Cette incidence varie de $38,46 \%$ à $84,61 \%$ sur les 13 tests effectués pour chaque extrait. 
Tableau 2. Résultats du criblage phytochimique

\begin{tabular}{|c|c|c|c|c|c|c|c|c|c|c|c|c|c|c|c|}
\hline & & \multicolumn{14}{|c|}{ Composés chimiques } \\
\hline $\begin{array}{c}\text { Noms / Familles } \\
\text { / parties } \\
\text { utilisées }\end{array}$ & Extraits & 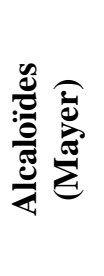 & 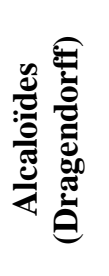 & 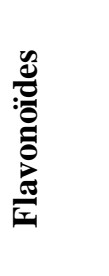 & 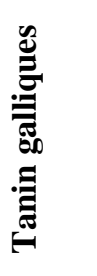 & 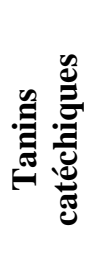 & 釆 & 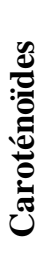 & : & $\stackrel{\mathscr{E}}{\mathscr{E}}$ & 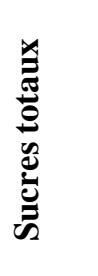 & 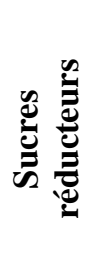 & 里 & 己ٌ & 绨 \\
\hline \multirow{4}{*}{$\begin{array}{c}\text { Cissus } \\
\text { Quadrangularis } \\
\text { (Linn) } \\
\text { Vitaceae / } \\
\text { feuilles }\end{array}$} & Cqf-EC & +++ & ++ & - & - & +++ & - & - & - & - & +++ & - & +++ & - & 38,46 \\
\hline & $\mathrm{Cq}_{\mathrm{f}-\mathrm{ED}}$ & +++ & +++ & - & - & +++ & - & - & - & - & +++ & - & +++ & - & 38,46 \\
\hline & Cqf-EM & +++ & +++ & +++ & +++ & - & - & - & - & - & +++ & +++ & +++ & - & 53,84 \\
\hline & $\mathbf{C q} \mathbf{f}-\mathbf{E A}$ & ++ & + & + & - & +++ & ++ & - & - & - & +++ & +++ & +++ & - & 61,54 \\
\hline \multirow{3}{*}{$\begin{array}{c}\text { Senna } \\
\text { Occidentalis } \\
\text { (Link) } \\
\text { Fabaceae / } \\
\text { feuilles }\end{array}$} & Sof-EC & +++ & +++ & + & - & +++ & - & - & +++ & - & +++ & - & + & - & 53,84 \\
\hline & Sof-ED & +++ & +++ & +++ & +++ & - & - & - & +++ & - & +++ & +++ & +++ & - & 61,54 \\
\hline & Sof-EM & +++ & +++ & +++ & +++ & - & +++ & - & +++ & ++ & +++ & +++ & +++ & +++ & 84,61 \\
\hline \multirow{4}{*}{$\begin{array}{c}\text { Cissus } \\
\text { Quadrangularis } \\
\text { (Linn) } \\
\text { Vitaceae / tiges }\end{array}$} & $\mathrm{Cq}_{\mathrm{t}}-\mathrm{EC}$ & ++ & +++ & + & - & ++ & - & + & - & - & +++ & - & + & +++ & 61,54 \\
\hline & $\mathrm{Cq} t-\mathrm{ED}$ & +++ & +++ & + & - & ++ & - & - & - & - & +++ & + & + & - & 53,84 \\
\hline & $\mathrm{Cq}$-EM & +++ & ++ & +++ & - & +++ & - & - & - & - & +++ & +++ & + & - & 53,84 \\
\hline & $\mathrm{Cq}_{\mathrm{t}}-\mathrm{EA}$ & - & + & - & - & + & - & - & - & + & +++ & +++ & + & - & 46,13 \\
\hline
\end{tabular}

La Figure 1 révèle une richesse qualitative en métabolites secondaires des plantes étudiées. Les alcaloïdes, les sucres totaux et les phénols sont les plus abondants, suivit des flavonoïdes, des tanins cathéchiques enfin, les sucres réducteurs et les saponosides. 


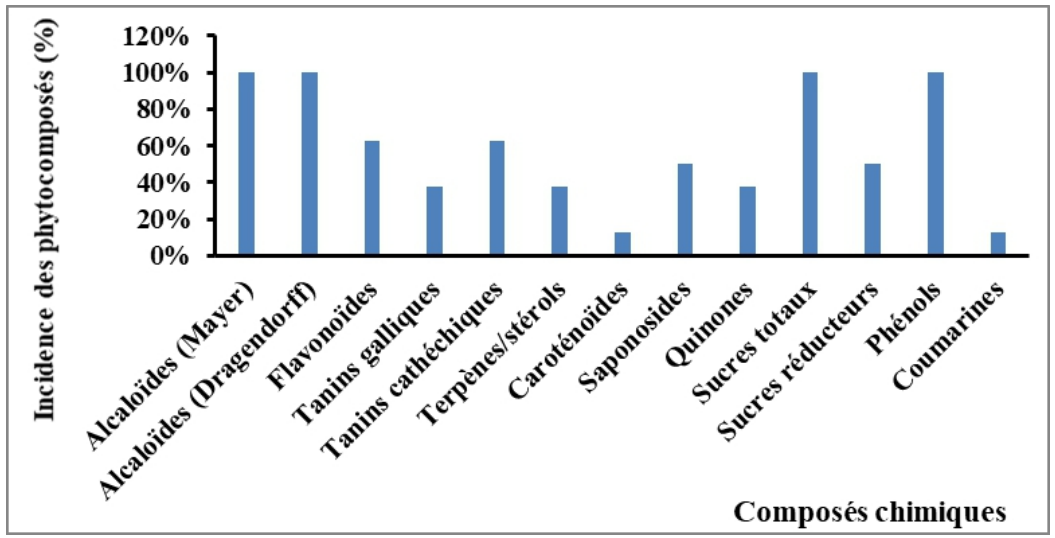

Figure 1. Histogramme des métabolites secondaires présents dans les extraits étudiés

\section{Incidence de la révélation de chaque métabolite secondaire dans les extraits}

L'incidence peut également être évaluée en regroupant chaque type de métabolites secondaires avérés positifs en fonction du nombre total d'extraits examinés (Figure 2). Dans le groupe A, les alcaloïdes, les phénols et les sucres totaux sont présents dans tous les 12 types d'extraits étudiés. Le groupe B révèle la présence des tanins cathéchiques dans 10 extraits. Les flavonoïdes et les sucres réducteurs présents dans 7 et 8 extraits respectivement forment le groupe $\mathrm{C}$. Dans le groupe $\mathrm{D}$, les saponosides sont présents dans 4 extraits puis les tanins galliques, les quinones, les terpènes ou les stérols sont présents dans 3 extraits. Les caroténoïdes et les coumarines sont quant à eux présents dans 2 extraits.

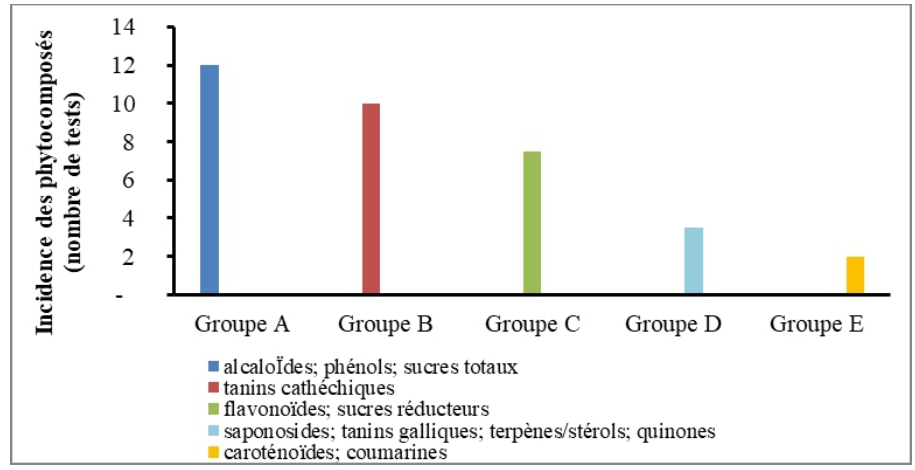

Figure 2. Histogramme représentant l'incidence de chaque métabolite secondaire dans les douze extraits étudiés

(Groupe A: métabolites révélés dans l'ensemble des extraits; Groupe B: métabolites révélés dans 10 extraits; Groupe $C$ : métabolites révélés dans 8 et/ou 7 extraits; Groupe D:

métabolites révélés dans 4 et/ou 3 extraits; Groupe E: métabolites révélés dans 2 extraits). 


\section{Répartition qualitative en fonction des solvants}

La richesse en composition chimique des extraits est également fonction du type de solvant (Figure 3). En effet, les extraits méthanoliques contiennent la majorité des composés phytochimiques examinés (84,61\% et 53,84\%). Suivi des extraits aqueux dans lesquelles deux ont une valeur de $61,54 \%$. Les extraits au cyclohéxanique et au dichlorométhanique s'équivalent avec des valeurs variant entre $61,54 \%$ et $38,46 \%$. Les feuilles de Senna occidentalis présentent une abondance en composition chimique comparé aux feuilles de Cissus quadrangularis.

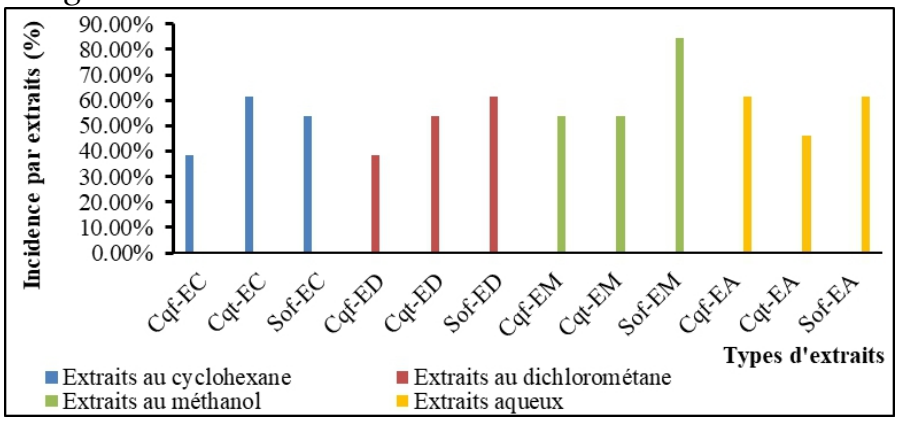

Figure 3. Histogramme représentant l'incidence des métabolites secondaires dans chaque extrait

\section{Discussions}

Le criblage phytochimique de Senna occidentalis (feuilles) et de Cissus quadrangularis (tige et feuilles) présente une richesse en métabolisme secondaire des extraits étudiés dans $l^{\text {'ensemble. }}$

Senna occidentalis est une plante utilisée en médecine traditionnelle au Gabon contre le ver de l'œil, comme fébrifuge, sudorifique, diurétique, antidiarrhée, blennorragique (Walker \& Sillans, 1961). Le criblage phytochimique de l'ensemble des extraits des feuilles révèle une abondance des sucres totaux, des alcaloïdes, des saponosides, des phénols et des flavonoïdes. Une faible présence des tanins, des stérols ou tritérpènes, des quinones, des sucres réducteurs et des coumarines. Les tanins galliques sont présent uniquement dans les extraits au dichlorométhane et au méthanol, tandis que les tanins cathéchiques sont présents dans les extraits au cychloheane et aqueux. Ces résultats sont en accord avec les travaux de Thiaw et al. (2015) qui ont montré que l'activité ovicide et/ou adulticide observée sur Caryedon serratus serait essentiellement due à l'action biologique des alcaloïdes, terpénoïdes, saponines ou flavonoïdes présents dans les différents extraits (éther de pétrole, méthanol, fraction hexane, fraction acétate d'éthyle et fraction méthanol) à partir des feuilles de $S$. occidentalis testés. Ces auteurs signalent que ces composés pourraient agir sur l'embryon ou sur la cuticule du ver adulte provoquant ainsi son asphysie. L'activité anthelmintique dose dépendante de l'extrait éthanolique de $S$. occidentalis a été révélé sur le ver adulte de 
Pheritima posthuma (Sayyad et al., 2014). Les extraits aqueux et hydroalcholiques de $S$. occidentalis ont présentés des activés larvicides et ovicides remarquables sur les œufs et les larves de Haemonchus contortus (Eguale et al., 2011). Un grand nombre de composés chimiques tels que les acaloïdes, anthocyanosides, phenoliques, protéines, phlobatanins, stéroïdes, tanins, flavonoïdes, anthroquinones, saponines, terpènes, résines, baumes, acides aminés, carbohydrates, sucres et glycosides cardiotoniques sont révélés sur différents extraits de S. occidentalis leur conférant ainsi plusieurs activités pharmacologiques (Al-Snafi, 2015). Bien qu'il n'y ait aucune étude faite sur la filaire $L$. loa, la richesse en métaboliste secondaire dans les quatre types d'extraits étudiés pourrait justifier l'usage en médecine traditionnelle de $S$. Occidentalis.

Cissus quadrangularis est une plante très connue sur le plan pharmacologique et phytochimique. Plusieurs propriétés pharmacologiques y ont été révélés (Mishra et al., 2010). Cependant, aucune étude n'a été faite sur la filaire Loa loa à ce jour. Au Gabon la tige sert de vulnéraire et la sève s'emploie pour combattre la filaire de l'œil en médecine traditionnelle (Walker \& Sillans, 1961). Le criblage phytochimique révèle également une variété de composants chimiques.

Très peu d'études ont été effectuées sur l'activité des plantes médicinales utilisées traditionnellement contre la filariose à Loa loa. Cependant, des extraits méthanoliques de neuf plantes gabonaises ont été testés sur Loa loa isolés du sang de patients infectés et ont révélé une activité filaricide promoteur de quatre extraits (Mengome et al., 2010). Dans la même étude, la composition phytochimique a révèle la prédominance des sucres réducteurs, des tanins, des polyphénols, des triterpènes, des saponosides et des alcaloïdes. Récemment, la voacangine et la voacamine, deux alcaloïdes isolés de l'écorce Voacanga africana inhibe l'activité de la Loa loa avec respectivement $5,49 \mu \mathrm{M}$ et $2,49 \mu \mathrm{M}$ de concentrations inhibitrices de 50 pour cent (Borakaeyebe et al., 2015). La littérature révèle que les métabolites secondaires purs ou modifiés, présenteraient plusieurs activités filaricides sur d'autres types de filaires. En effet, l'extrait éthanolique des tiges de Lantana camara a montré une activité microfilaricide à 43,05\% par voie orale sur des rongeurs de type Mastomys coucha infestés par Brugia malayi, une filariose Lymphatique humaine et sur Acanthocheilonema viteae une filariose de rongeur à 95,04\% et stérilise les femelles à 60,66\% avec une action macrofilaricide légère (Misra et al., 2007). L'activité macrofilaricide et microfilaricide in vitro et in vivo sur Brugia malayi a été révélée dans l'extrait éthanolique ainsi que dans la fraction hexanique de Calotropis procera (Kushwaha et al., 2019). L'extrait méthanolique des racines de Vitex negundo riche en alcaloïdes, saponosides et flavonoïdes et l'extrait méthanolique des feuilles d'Aegle marmelos riche en coumarines, révèlent une activité 
microfilaricide sur Brugia malayi (Sahara et al., 2008). Les sucres qui sont des molécules de base chez les plantes, ils ont également montré une activité macrofilaricide [Chatterjee et al., 1992]. Les galactolipides sucres isolés sur Bauhinia racemose révèlent une activité filaricide contre Brugia malayi (Sashidhara et al., 2012). Les monoterpènes phénoliques isolés de l'extrait méthanolique des fruits de Trachyspermum ammi présenteraient une activité macrofilaricide sur Setaria digitata une filariose bovine puis sur Brugia malayi et stérilise les femelles (Mathew et al., 2008). Un certain nombre de composés phytochimiques (linalol, alpha-pinène, strychnine, vanilline, pipérine, isoeugénol, curcumine, bêta-caryophyllène, acide cinnamique, capsaïcine, citronellol, géraniol) seraient des efficaces inhibiteurs de la Glutathion-S-transférase (GST) de Brugia malayi, enzyme de détoxification de ce filaire et pourraient servir de base pour des futurs médicaments contre les nématodes filariens (Azeez et al., 2012). La richesse en composés phytochimiques de Senna occidentalis et de Cissus quadrangularis reste une voie à explorer afin de caractériser la ou les molécules et de prouver leurs activités toxicologiques et pharmacologiques sur la filariose à Loa loa.

\section{Conclusion}

Les analyses phytochimiques réalisées sur quatre échantillons issus de deux plantes ont permis d'estimer leur richesse en phytocomposés. Le criblage phytochimique de Senna occidentalis et de Cissus quadrangularis a permis de détecter un ensemble de métabolites secondaires tels que les sucres totaux, les alcaloïdes, les flavonoïdes, les tanins, les triterpènes/stérols, les caroténoïdes, les quinones, les coumarines et les saponosides. Des investigations sur les activités filaricides à Loa loa ainsi que les effets d'innocuités de molécules ou de solutions efficaces, accessibles sont nécessaires.

\section{References:}

1. Agbolade, O.M., Akinboye, D.O. \& Ogunkolo, O. (2005). Loa loa and Mansonella perstans: Neglected human infections that need control in Nigeria. African Journal of Biotechnology, 4(13), 1554-1558.

2. Akué, J.P., Nkoghe, D., Padilla, C., Moussavou, G., Moukana, H., Mbou, R.A., Ollomo, B. \& Leroy, E. M. (2011). Epidemiology of concomitant infection due to Loa loa and Mansonella perstans in Gabon. PloS Negleted Tropical Diseases, 5(10), e1329:1-11.

3. Al-Snafi, A. E. (2015). The therapeutic impotance Cassia occidentalis - An overview. Indian Journal of Pharmaceutical Science \& Research, 5(3), 158-171.

4. Antinori, S., Schifanella, L., Million, M., Galimberti, L., Ferraris, L., Mandia, L., Trabucchi, G., Cacioppo, V., Manaco, G., Tosoni, A., 
Brouqui, P., Gismondo, M. R., Giuliani, G. \& Corbellino, M. (2012). Imported Loa loa flariasis: Three cases and a review of cases reported in non-endemic countries in the past 25 years. International Journal of Infectious Diseases, 16(9), e649-662.

5. Azeez, S., Babu, R.O., Aykkal, R. \& Narayanan, R. (2012). Virtual screening and in vitro assay of potential drug like inhibitors from spices against glutathione-S-transferase of filarial nematodes. Journal of Molecular Modeling, 18(1), 151-163.

6. Behera, D.R. \& Bhatnagar, S. (2018). Filariasis: Role of medicinal plant in lymphatic filariasis. International Journal of Herbal Medicine, 6(1), $40-46$.

7. Borakaeyabe, S.B., Mbah, J.A., Cho-Ngwa, F., Metuge, J.A. \& Mbua Ngale Efange, S. (2015). Isolation and characterization of filaricidal compounds from the stem bark of Vocanga africana, a plant used in the traditional treatment of onchocerciasis in Cameroon. Journal of Medicine Plants Research, 9(14), 471- 478.

8. Boussinesq, M. (2006). Loiasis. Annals of Tropical Medicine Parasitology 100(8), 715-731.

9. Chatterjee, R. K., Fatma, N., Murthy, P. K., Sinha, P., Kulshrestha, D. K. \& Dhawan, B. N. (1992). Macrofilaricidal activity of the stem bark of Streblus asper and its major active constituents. Drug Development Research, 26(1), 67-78.

10. Chippaux, J. P., Boussinesq, M., Gardon, J., Gardon-Wendel, N. \& Emould, J. C. (1996). Severe adverse reaction risks during mass treatment with ivermectin in loiasis-ndemic areas. Parasitology Today, 12(11), 448-450.

11. Eguale, T., Tadesse, D. \& Giday, M. (2011). In vitro anthelmintic activity of crude extracts of five medicinal plants against egg- hatching and larval development of Haemonchus contortus. Journal of Ethnopharmacology, 137(1), 108-113.

12. Fain, A. (1981). Epidémiologie et pathologie de la loase. Annales de la société Belge de Médecine Tropicale, 61, 277-285

13. Feuya Tchouya, R. G., Souza, A., Tchouankeu, J. C., Yala, J. F., Boukandou, M., Foundikou, H., Obiang, G. D., Boyom, F. F., Mabika, R. M., Menkem, E. Z., Ndinteh, D. T. \& Lebibi, J. (2015). Ethnopharmacological surveys and pharmacological studies of plants used in traditional medicine in the treatment of HIV / AIDS opportunistic diseases in Gabon. Journal of Ethnopharmacology. 162, 306-316.

14. Kushwaha, V., Rastogi, S., Pandey, M. M., Saxena, K., Khatoon, S., Rawat, A. K. S. \& Murthy, P. K. (2019). In Vitro and In Vivo Antifilarial Activity of Standardized Extract of Calotropis procera 
Flowers against Brugia malayi. Current Topics in Medicinal Chemistry. 19(14):1252-1262.

15. Lengani, A., Lompo, L. F., Guissou, I. P. \& Nikiema, J. P. (2010). Médecine traditionnelle et maladies des reins au Burkina Faso. Néphrologie et Thérapeutique, 6(1), 35-39.

16. Mathew, N., Misra-Bhattacharya, S., Perumal, V. \& Muthuswamy, K. (2008). Antifilarial lead molecules isolated from Trachyspermum ammi. Molecules, 13(9), 2156-2168.

17. Mengome, L. E., Feuya Tchouya, G. R, Eba, F. \& Nsi-Emvo, E. (2009). Antiproliferative effect of alcoholic extracts of some Gabonese medicinal plants on human colonic cancer cells. African Journal Traditional Complementary Alternative Medicines. 6(2), 112-117.

18. Mengome, L. E., Akué, J. P., Souza, A., Feuya Tchoua, G. R. \& Nsi Emvo, E. (2010). In vitro activities of plant extracts on human Loa loa isolates and cytotoxicity for eukaryotic cells. Parasitology Research, 107(3), 643-50.

19. Mishra, G., Srivastava, S. \& Nagori, B. P. (2010). Pharmacological and Therapeutic Activity of Cissus quadrangularis : An Overview. International Journal, 2(2), 1298-1310.

20. Misra, N., Sharma, M., Raj, K., Dangi, A., Srivastava, S. \& MisraBhattacharya, S. (2007). Chemical constituents and antifilarial activity of Lantana camara against human lymphatic filariid Brugia malayi and rodent filariid Acanthocheilonema viteae maintained in rodent hosts. Parasitology Research. 100(3), 439-448.

21. Moussala, M., Fobi, G., Zogo, O., Hiag, B. L. A., Bengono, G. \& McMoli, T. E. (2004). Survenue d'hémorragies rétiniennes lors du traitement de l'onchocercose par l'invermectine chez une patiente coinfectée par la loase. Journal Français d'Ophtalmologie. 27(1), 63-66.

22. Ndjonka, D., Rapado, L. N., Silber, A. M. \& Liebau, E. (2013). Natural Products as a Source for Treating Neglected Parasitic Diseases. International Journal of Molecular Sciences. 14(2), 3395-3439.

23. Newman, D. J. \& Cragg, G. M. (2007). Natural products as sources of new drugs over the last 25 years. Journal of Natural Products. 70(3), $461-477$.

24. Organisation Mondiale de la Santé. Programme mondial pour l'élimination de la filariose lymphatique: rapport de situation (2018). REH 2019; 94: 457-470.

25. Pawlaczyk, I., Capek, P., Czerchawski, L., Bijak, J., Lewik-Tsirigotis, M., Pliszczak-Król, A. \& Gancarz, R. (2011). An anticoagulant effect and chemical characterization of Lythrum salicaria L. glycoconjugates. Carbohydrate Polym, 86; 277-284. 
26. Sahare, K. N., Anandhraman, V., Meshram, V. G., Meshram, S. U., Reddy, M. V., Tumane, P. M. \& Goswami, K. (2008). Antimicrofilarial activity of methanolic extract of Vitex negundo and Aegle marmelos and their phytochemical analysis. Indian Journal Experimental Biology. 46(2), 128-131.

27. Sashidhara, K. V., Singh, S. P., Misra, S., Gupta, J. \& MisraBhattacharya, S. (2012). Galactolipids from Bauhinia racemosa as a new class of antifilarial agents against human lymphatic filarial parasite, Brugia malayi. European Journal of Medicinal Chemistry. 50, 230-235.

28. Sayyad, R. R., Kare, R. D., Jagtap, S. M., Katkar, S. T. \& Kadam, J. H. (2014). Anthelmintic activity of ethanolic extract of Cassia Occidentalis Linn. International Journal of Pharmacy Research Sciences. 2(1), 42-46.

29. Thiaw, C., Coly, E. V., Djiba, S., Diop, M., Ndoye, O., Cisse, N. \& Semben, M. (2015). Senna occidentalis L., une plante prometteuse dans la lutte contre Caryedon serratus Ol. (Coleoptera, Bruchidae), insecte ravageur des stocks d'arachide au Sénégal. International Journal of Biological and Chemical Sciences. 9(3), 1399 -1418.

30. Walker, A. \& Sillans, R. (1961). Les plantes utiles du Gabon. Encyclopédie Biologique 56. Paris: Paul Lechevalier. p. 614.

31. Zouré, H. G., Wanji, S., Noma, M., Amazigo, U. V., Diggle, P. J., Tekle, A. H. \& Remme J. H. (2011). The geographic distribution of Loa loa in Africa: Results of large-scale implementation of the rapid assessment procedure for loiasis (RAPLOA). PLoS Negleted Tropical Diseases. 5(6), e1210. 